JEAN B. LASSERRE (Toulouse)

Eduardo S. Zeron (México, D.F.)

\title{
SOLVING A CLASS OF MULTIVARIATE INTEGRATION PROBLEMS VIA LAPLACE TECHNIQUES
}

Abstract. We consider the problem of calculating a closed form expression for the integral of a real-valued function $f: \mathbb{R}^{n} \rightarrow \mathbb{R}$ on a set $S$. We specialize to the particular cases when $S$ is a convex polyhedron or an ellipsoid, and the function $f$ is either a generalized polynomial, an exponential of a linear form (including trigonometric polynomials) or an exponential of a quadratic form. Laplace transform techniques allow us to obtain either a closed form expression, or a series representation that can be handled numerically. Finally, a methodology is proposed for multivariate functions $f$ which have a (multidimensional) Laplace transform.

1. Introduction. The main goal of this work is to calculate a closed form for the integral

$$
\int_{S} f(x) d x
$$

when the sets $S \subset \mathbb{R}^{n}$ are either convex polyhedra $\left\{x \in \mathbb{R}^{n} \mid A x \leq b\right\}$ or ellipsoids $\left\{x \in \mathbb{R}^{n} \mid x^{\prime} A x \leq b\right\}$, and the real-valued functions $f: \mathbb{R}^{n} \rightarrow \mathbb{R}$ belong to a certain class.

This kind of multivariate integrals are classically calculated (numerically) using finite element methods (see e.g. Zienkiewicz and Taylor [9]). That is, one decomposes $S$ into elementary cells (in particular, simplices) where the function $f$ is suitably approximated by polynomials and integrated via quadrature formulae. The case of ellipsoids is interesting in some applications. For instance, given a random vector $X$ with values in $\mathbb{R}^{n}$, one

2000 Mathematics Subject Classification: 65D30, 65D15, 65R10.

Key words and phrases: multivariate integration, Laplace transform.

Research partially supported by the ECOS-Nord (France)-ANUIES (México) Educational and Scientific Cooperation Program PM98M02. The second author wishes to thank Cinvestav-IPN (México) for its support. 
may wish to compute the probability $P(X \in S)$ that $X$ belongs to $S$, especially when $S$ is a compact set far away from the mean $\bar{X}$, let us say. An ellipsoid centered at some $x_{0}$ away from $\bar{X}$ is a convenient example of such a set $S$.

Even if the use of Laplace transform is not new in deriving closed form expressions for univariate integration, its use for multivariate integration seems not so well developed. Some of the results presented in this paper are not new, but to our knowledge they were obtained with other techniques: mainly inductive arguments, and in some cases Stokes' theorem as well. The reader will see that in many interesting cases the Laplace transform yields short and direct proofs with no induction argument. The main reason is that the product of two (elementary) Laplace transforms can often be inverted directly, avoiding in this way the convolution (the latter is in many cases just a rephrasing of the original multivariate integral into a convolution of one-dimensional integrals).

The class of functions considered here is invariant under affine transformations. Thus, in the case of a convex polyhedron (which can be partitioned into elementary simplices), we may restrict our analysis to the canonical simplex $\Delta(z)=\left\{x \in \mathbb{R}_{+}^{n}: \sum_{i} x_{i} \leq z\right\}$. Similarly, for an ellipsoid, we may also restrict to the generic $n$-ball $S(z):=\left\{\|x\|^{2} \leq z\right\}$. We then consider the integral in (1.1) as a function of the single parameter $z$, and we calculate its Laplace transform. Sometimes the inversion of this Laplace transform can be done directly or via a real series inversion technique described in Widder [8]. In the latter case, it is important to emphasize that the problem is reduced to computing a series expansion of a univariate function, as opposed to the multivariate original problem in $\mathbb{R}^{n}$. Moreover, the convergence of the resulting series is usually faster in view of the coefficients introduced in the real inversion technique.

We also obtain, as a by-product, a series representation of $f(x(z))$ in the mean value theorem $\int_{S} f(x) d x=\operatorname{vol}(S) f(x(z))$ with $S$ being $\Delta(z)$ or $S(z)$.

We finally consider the case of arbitrary functions $f: \mathbb{R}^{n} \rightarrow \mathbb{R}$ whose multi-dimensional Laplace transform $F: \mathbb{C}^{n} \rightarrow \mathbb{C}$ exists. Following the same techniques, if the function $F\left(\lambda e_{n}\right)$ (with $e_{n}$ the unit vector $(1, \ldots, 1)$ of $\left.\mathbb{R}^{n}\right)$, evaluated in the bisector $\lambda_{j}=\lambda$ for all $j$, has a Laurent series expansion in $\lambda^{-1}$, then the integral of $f$ over the simplex $\Delta(z)$ can be expressed as a power series in terms of $z$ whose coefficients can be easily deduced from the series expansion of $F\left(\lambda e_{n}\right)$. That is, the original $n$-dimensional problem reduces to a one-dimensional series inversion, provided the Laplace transform $F$ is known.

This paper is organized as follows: We firstly integrate on a convex polyhedron $\left\{x \in \mathbb{R}^{n} \mid A x \leq b\right\}$. Subsequently, we analyse the integral on an ellipsoid $\left\{x \in \mathbb{R}^{n} \mid x^{\prime} A x \leq b\right\}$, when $f$ is a generalized polynomial, an ex- 
ponential of a linear form or an exponential of a quadratic function. The last section is devoted to integrating arbitrary functions $f$, whose Laplace transform is available, on the simplex $\Delta(z)$.

2. Integration on a convex polyhedron. Consider the problem (1.1) with $S:=\left\{x \in \mathbb{R}^{n} \mid A x \leq b\right\}$ for a given matrix $A \in \mathbb{R}^{m \times n}$ and vector $b \in \mathbb{R}^{m}$. We assume that $S$ is compact in order to decompose it into elementary simplices $\left\{\Delta_{i}\right\}$, that is, $\bigcup_{i} \Delta_{i}=S$, so it suffices to consider the problem (1.1) with $S:=\Delta_{i}$.

Moreover, let $\left\{x_{0}, x_{1}, \ldots, x_{n}\right\}$ be the vertices of $\Delta_{i}$. Making the change of variable $x=x_{0}+\sum_{i=1}^{n} y_{i}\left(x_{i}-x_{0}\right)$, we obtain

$$
\int_{\Delta_{i}} f(x) d x=\operatorname{vol}\left(\Delta_{i}\right) \int_{\Delta(1)} g(y) d y
$$

where

$$
y \mapsto g(y):=f\left(x_{0}+\sum_{i=1}^{n} y_{i}\left(x_{i}-x_{0}\right)\right),
$$

and $\Delta(1)$ is the canonical simplex (with $z:=1$ )

$$
z \mapsto \Delta(z):=\left\{y \in \mathbb{R}^{n} \mid \sum_{i=1}^{n} y_{i} \leq z ; y_{i} \geq 0, i=1, \ldots, n\right\}, \quad z \in \mathbb{R}^{+} .
$$

Thus, we can address problem (1.1) fixing $S$ to be the simplex $\Delta(z)$ given in $(2.1)$.

2.1. Generalized polynomials. Consider the case when $f: \mathbb{R}^{n} \rightarrow \mathbb{R}$ is given by

$$
x \mapsto f(x)=\prod_{i=1}^{n} x_{i}^{\alpha_{i}}, \quad \alpha_{i} \in \mathbb{R}, i=1, \ldots, n ;
$$

and more generally, when $f$ is a sum of such functions. Observe that we do not require $\alpha_{i}$ to be integers. Let $\Gamma(\cdot)$ be the gamma-function defined by the Euler product expansion

$$
\frac{1}{\Gamma(1-s)}:=\lim _{n \rightarrow \infty} n^{s} \prod_{k=1}^{n}\left(1-\frac{s}{k}\right) .
$$

TheOrem 2.1. Let $f: \mathbb{R}^{n} \rightarrow \mathbb{R}$ be as in (2.2) and $\Delta(z)$ be as in (2.1). Assume that $\alpha_{i}>-1$ for all $i=1, \ldots, n$. Then

$$
\begin{aligned}
\int_{\Delta(z)} f(x) d x & =\frac{\prod_{i=1}^{n} \Gamma\left(\alpha_{i}+1\right)}{\Gamma\left(n+1+\sum_{i=1}^{n} \alpha_{i}\right)} z^{n+\sum_{i=1}^{n} \alpha_{i}} \\
& =\operatorname{vol}(\Delta(z)) \frac{\Gamma(1+n) \prod_{i=1}^{n} \Gamma\left(\alpha_{i}+1\right)}{\Gamma\left(n+1+\sum_{i=1}^{n} \alpha_{i}\right)} z^{\sum_{i=1}^{n} \alpha_{i}}
\end{aligned}
$$


Proof. Let $g: \mathbb{R}^{+} \rightarrow \mathbb{R}^{+}$be the function

$$
y \mapsto g(y):=\int_{\Delta(y)} f(x) d x
$$

and let $G: \mathbb{C} \rightarrow \mathbb{C}$ be its Laplace transform, that is,

$$
\lambda \mapsto G(\lambda):=\int_{0}^{\infty} \mathrm{e}^{-\lambda y}\left[\int_{x \geq 0, \sum_{i} x_{i} \leq y} f(x) d x\right] d y, \quad \lambda \in \mathbb{C}, \Re(\lambda)>0,
$$

where $\Re(\lambda)$ stands for the real part of $\lambda$. By Fubini's theorem (valid here),

$$
\begin{aligned}
G(\lambda) & =\int_{x \geq 0} f(x)\left[\int_{\sum_{i} x_{i} \leq y} \mathrm{e}^{-\lambda y} d y\right] d x \\
& =\frac{1}{\lambda} \int_{x \geq 0} \mathrm{e}^{-\lambda \sum_{i} x_{i}} f(x) d x=\frac{1}{\lambda} \prod_{i=1}^{n} \int_{0}^{\infty} \mathrm{e}^{-\lambda x_{i}} x_{i}^{\alpha_{i}} d x_{i} \\
& =\lambda^{-1-n-\sum_{i=1}^{n} \alpha_{i}} \prod_{i=1}^{n} \Gamma\left(\alpha_{i}+1\right) \\
& =\frac{\Gamma\left(1+n+\sum_{i=1}^{n} \alpha_{i}\right)}{\lambda^{\left(1+n+\sum_{i=1}^{n} \alpha_{i}\right)}} \cdot \frac{\prod_{i=1}^{n} \Gamma\left(\alpha_{i}+1\right)}{\Gamma\left(1+n+\sum_{i=1}^{n} \alpha_{i}\right)} .
\end{aligned}
$$

We recognize the Laplace transform of $z \mapsto z^{n+\sum_{i=1}^{n} \alpha_{i}}$ in the first term of the last equality. The result follows.

The result in the case of integer coefficients $\left\{\alpha_{i}\right\}$ is known (see e.g. Stroud [6]). An explicit formula in terms of the vertices is given in Lasserre and Avrachenkov [4] for homogeneous polynomials on an arbitrary simplex $\Delta$, and using a different technique. An immediate consequence of Theorem 2.1 is the possibility of integrating analytic functions. For illustration and to avoid heavy notation, we restrict ourselves to $\mathbb{R}^{2}$, but the result easily extends to arbitrary dimension.

Corollary 2.2. Let $f: \mathbb{R}^{2} \rightarrow \mathbb{R}$ be analytic, that is,

$$
x \mapsto f(x)=\sum_{n=0}^{\infty}\left[\sum_{k=0}^{n} a_{n k} x_{1}^{k} x_{2}^{n-k}\right], \quad x \in \mathbb{R}^{2},
$$

where the series converges uniformly on compact subsets of $\mathbb{R}^{2}$. Then

$$
\begin{aligned}
\int_{\Delta(z)} f(x) d x & =\sum_{n=0}^{\infty} \frac{z^{n+2}}{(n+2) !}\left[\sum_{k=0}^{n} a_{n k} k !(n-k) !\right] \\
& =\operatorname{vol}(\Delta(z)) \sum_{n=0}^{\infty} \frac{z^{n}}{\left(\begin{array}{c}
n+2 \\
2
\end{array}\right)}\left[\sum_{k=0}^{n} \frac{a_{n k}}{\left(\begin{array}{c}
n \\
k
\end{array}\right)}\right] .
\end{aligned}
$$


Proof. Just integrate each term of the series (2.9) on $\Delta(z)$ and apply Theorem 2.1.

If $f$ is not analytic, but has a series expansion like (2.9) with real exponents (for instance $f(x, y)=\sqrt{x y} g(x, y)$ with $g$ analytic) larger than -1 , then an analog of Corollary 2.2 also holds with obvious adjustments.

2.2. Exponentials of linear type. We could apply Corollary 2.2 to integrate multivariate exponential functions, since they are analytic. However, we may also proceed directly to get an expression in closed form.

Consider the case where $f: \mathbb{R}^{n} \rightarrow \mathbb{R}$ is given by

$$
x \mapsto f(x):=\mathrm{e}^{\langle c, x\rangle}
$$

for some $c \in \mathbb{R}^{n}$ with $c=\left\{c_{1}, \ldots, c_{n}\right\}$. We need to define $c_{0}:=0$ as well. In fact, we will see later that we can also consider $c \in \mathbb{C}^{n}$ to be a purely imaginary number $c:=a \sqrt{-1}$ with $a \in \mathbb{R}^{n}$; this construction will allow us to handle trigonometric polynomials.

Theorem 2.3. Let $f: \mathbb{R}^{n} \rightarrow \mathbb{R}$ be as in (2.12) and $\Delta(z)$ be as in (2.1). Assume that $c_{i} \neq c_{j}$ for all $i \neq j$. Then

$$
\begin{aligned}
\int_{\Delta(z)} f(x) d x & =\sum_{i=0}^{n} \frac{\mathrm{e}^{c_{i} z}}{\prod_{j \neq i}\left(c_{i}-c_{j}\right)} \\
& =\operatorname{vol}(\Delta(z)) \sum_{i=0}^{n} \frac{\mathrm{e}^{c_{i} z} n !}{\prod_{j \neq i}\left(c_{i} z-c_{j} z\right)} .
\end{aligned}
$$

Proof. Again, let $g: \mathbb{R}^{+} \rightarrow \mathbb{R}^{+}$be as in (2.6) and let $G: \mathbb{C} \rightarrow \mathbb{C}$ as in (2.7) be its Laplace transform. Straightforward calculation with a Fubini argument yields

$$
G(\lambda)=\prod_{i=0}^{n}\left(\lambda-c_{i}\right)^{-1}, \quad \Re(\lambda)>\max _{i} c_{i},
$$

which is the Laplace transform of

$$
z \mapsto \sum_{i=0}^{n} \frac{\mathrm{e}^{c_{i} z}}{\prod_{j \neq i}\left(c_{i}-c_{j}\right)} ;
$$

this yields (2.13). Then (2.14) follows from $\operatorname{vol}(\Delta(z))=z^{n} / n !$.

A similar result for the $(n-1)$-simplex $\left\{x \in \mathbb{R}_{+}^{n} \mid \sum_{i=1}^{n} x_{i}=1\right\}$ was demonstrated in Barvinok [1] using the Fourier transform of the function $\left.\mathrm{e}^{\langle c, x\rangle}\right|_{x \in \mathbb{R}_{+}^{n}}$ (with $c<0$ ) and induction on the dimension in Podkorytov [2]. A formula for integrating $\mathrm{e}^{\langle c, x\rangle}$ over a convex polytope was proved in [1] via Stokes' theorem, and in Lasserre [3] with different arguments. The above result shows that the Laplace transform technique yields a very short proof. 
Trigonometric polynomials. Notice that equation (2.13) is still valid if we let $c \in \mathbb{C}^{n}$ be a complex vector. In particular, we can handle trigonometric polynomials by setting $c=a \sqrt{-1}$ with $a \in \mathbb{R}^{n}$. For example, consider the case when $x \mapsto f(x):=\sin \langle a, x\rangle, x \in \mathbb{R}^{n}$. We can apply Theorem 2.3 with $c:=a \sqrt{-1}$, provided that $a_{i} \neq a_{j}$ for all $i \neq j$ and $a_{0}:=0$, because $\int_{\Delta(z)} \sin \langle a, x\rangle d x=\Im\left(\int_{\Delta(z)} \mathrm{e}^{\langle a, x\rangle \sqrt{-1}} d x\right)$. Moreover, we have

COROLlary 2.4. Let $a \in \mathbb{R}^{n}$ be such that $a_{i} \neq a_{j} \neq 0$ for all $i \neq j$. Then, with $n=2 p+1$,

$$
\begin{gathered}
\int_{\Delta(z)} \sin \langle a, x\rangle d x=(-1)^{p} \sum_{i=0}^{n} \frac{-\cos \left(a_{i} z\right)}{\prod_{j \neq i}\left(a_{i}-a_{j}\right)}, \\
\int_{\Delta(z)} \cos \langle a, x\rangle d x=(-1)^{p} \sum_{i=0}^{n} \frac{\sin \left(a_{i} z\right)}{\prod_{j \neq i}\left(a_{i}-a_{j}\right)},
\end{gathered}
$$

and with $n=2 p$,

$$
\begin{aligned}
& \int_{\Delta(z)} \sin \langle a, x\rangle d x=(-1)^{p} \sum_{i=0}^{n} \frac{\sin \left(a_{i} z\right)}{\prod_{j \neq i}\left(a_{i}-a_{j}\right)}, \\
& \int_{\Delta(z)} \cos \langle a, x\rangle d x=(-1)^{p} \sum_{i=0}^{n} \frac{\cos \left(a_{i} z\right)}{\prod_{j \neq i}\left(a_{i}-a_{j}\right)} .
\end{aligned}
$$

REMARK 2.5. If $\Delta(1)$ is obtained by an affine transformation from an arbitrary simplex $\Delta$ with vertices $x_{0}, x_{1}, \ldots, x_{n}$, then formula (2.13) (and formulas (2.15)-(2.18)) can be rewritten as a compact formula in terms of the vertices $\left\{x_{i}\right\}$. Namely, (2.13) becomes

$$
\begin{aligned}
\int_{\Delta} f(x) d x & =\operatorname{vol}(\Delta) \int_{\Delta(1)} \mathrm{e}^{\left\langle c, x_{0}\right\rangle} \mathrm{e}^{\sum_{i=1}^{n} y_{i}\left\langle c, x_{i}-x_{0}\right\rangle} d y \\
& =\operatorname{vol}(\Delta) \sum_{i=0}^{n} \frac{\mathrm{e}^{\left\langle c, x_{i}\right\rangle}}{\prod_{j \neq i}\left\langle c, x_{i}-x_{j}\right\rangle},
\end{aligned}
$$

and, for instance, with $n=2 p+1$, (2.15) becomes

$$
\int_{\Delta} \sin \langle a, x\rangle d x=\operatorname{vol}(\Delta)(-1)^{p} \sum_{i=0}^{n} \frac{-\cos \left\langle a, x_{i}\right\rangle}{\prod_{j \neq i}\left\langle a, x_{i}-x_{j}\right\rangle}
$$

2.3. Exponentials of Gaussian type. Now we consider $f: \mathbb{R}^{n} \rightarrow \mathbb{R}$ defined by

$$
x \mapsto f(x):=\mathrm{e}^{x^{\prime} Q x},
$$

for some real-valued symmetric matrix $Q \in \mathbb{R}^{n \times n}$. Let $H_{k}: \mathbb{R}^{n \times 2 k} \rightarrow \mathbb{R}$ be the $2 k$-linear form associated with the homogeneous polynomial $\left(x^{\prime} Q x\right)^{k}$. 
That is, for instance, with $n=2$,

$$
\begin{aligned}
H_{1}\left(X_{1}, X_{2}\right)= & X_{1}^{\prime} Q X_{2}, \\
H_{2}\left(X_{1}, X_{2}, X_{3}, X_{4}\right)= & \frac{1}{3}\left[X_{1}^{\prime} Q X_{2} X_{3}^{\prime} Q X_{4}+X_{1}^{\prime} Q X_{3} X_{2}^{\prime} Q X_{4}\right. \\
& \left.+X_{1}^{\prime} Q X_{4} X_{2}^{\prime} Q X_{3}\right], \quad \text { etc. }
\end{aligned}
$$

Theorem 2.6. Let $\Delta$ be an $n$-simplex with vertices $\left\{a_{0}, \ldots, a_{n}\right\}$. Then

$$
\int_{\Delta} \mathrm{e}^{x^{\prime} Q x} d x=\operatorname{vol}(\Delta) \sum_{k=0}^{\infty} \frac{A_{k}}{k !}
$$

with

$$
A_{k}=\frac{1}{\left(\begin{array}{c}
n+2 k \\
2 k
\end{array}\right)} \sum_{0 \leq i_{1} \leq \ldots \leq i_{2 k} \leq n} H_{k}\left(a_{i_{1}}, \ldots, a_{i_{2 k}}\right) .
$$

Proof. The generic term in the series expansion of the exponential $\mathrm{e}^{x^{\prime} Q x}$ is $\left(x^{\prime} Q x\right)^{k} / k$ !, or equivalently $H_{k}(x, \ldots, x)$, a homogeneous polynomial of degree $2 k$. As the series of $\mathrm{e}^{x^{\prime} Q x}$ converges uniformly on compact subsets of $\mathbb{R}^{n}$, we can integrate each term over $\Delta$ and sum up. From a result in Lasserre and Avrachenkov [4],

$$
\int_{\Delta} H_{k}(x, \ldots, x) d x=\frac{\operatorname{vol}(\Delta)}{\left(\begin{array}{c}
n+2 k \\
2 k
\end{array}\right)} \sum_{0 \leq i_{1} \leq \ldots \leq i_{2 k} \leq n} H_{k}\left(x_{i_{1}}, \ldots, x_{i_{2 k}}\right),
$$

which yields (2.21).

In fact, this result is valid for all analytic functions $f: \mathbb{R}^{n} \rightarrow \mathbb{R}$, and extends Corollary 2.2 to arbitrary simplices.

3. Integration on an ellipsoid. Let $S$ be any ellipsoid $\left\{(x-a)^{\prime} Q(x-a)\right.$ $\leq z\}$ for a given real-valued symmetric positive definite matrix $Q \in \mathbb{R}^{n \times n}$ and real number $z \in \mathbb{R}^{+}$. We may assume, after applying an affine transformation, that in fact

$$
S=S(z):=\left\{x \in \mathbb{R}^{n} \mid \sum_{i=1}^{n} x_{i}^{2} \leq z\right\}
$$

is an $n$-ball of radius $z^{1 / 2}$.

Indeed, in many cases, the integration of a function $f$ over an arbitrary ellipsoid reduces, after an affine transformation, to the integration of a function $g$ over an $n$-ball, with $g$ still in a class of (nice) functions studied below.

3.1. Generalized polynomials. Integrating a homogeneous polynomial $x \mapsto p(x):=\prod_{i=1}^{n} x_{i}^{\alpha_{i}}$ on $S(z)$ is trivial if some exponent $\alpha_{i}$ is an odd 
integer. Indeed, assume that $\alpha_{1}$ is odd. Then

$$
\int_{S(z)} p(x) d x=\int_{-z^{1 / 2}}^{z^{1 / 2}} x_{1}^{\alpha_{1}}\left[\int_{\sum_{j=2}^{n} x_{j}^{2} \leq z-x_{1}^{2}} \prod_{j=2}^{n} x_{j}^{\alpha_{j}} d x_{2} d x_{3} \ldots d x_{n}\right] d x_{1}
$$

vanishes. Thus, nontrivial cases appear only when each exponent $\alpha_{i}$ is an even integer, and in this case we can even write $p(x)=\prod_{i=1}^{n}\left|x_{i}\right|^{\alpha_{i}}$. We will integrate here the generalized polynomial

$$
x \mapsto f(x)=\prod_{i=1}^{n}\left|x_{i}\right|^{\alpha_{i}}, \quad x \in \mathbb{R}^{n},
$$

where each $\alpha_{i} \in \mathbb{R}$ is not necessarily an integer.

TheOrem 3.1. Let $f: \mathbb{R}^{n} \rightarrow \mathbb{R}$ be as in (3.2) and $S(z)$ as in (3.1). Assume moreover that $\alpha_{i}>-1$ for all $i=1, \ldots, n$. Then

$$
\begin{aligned}
\int_{S(z)} f(x) d x & =2^{-n} \frac{\prod_{i=1}^{n} \Gamma\left(\frac{\alpha_{i}+1}{2}\right)}{\Gamma\left(1+\left(n+\sum_{i=1}^{n} \alpha_{i}\right) / 2\right)} z^{\left(n+\sum_{i} \alpha_{i}\right) / 2} \\
& =\operatorname{vol}(S(z)) \frac{\Gamma(1+n / 2) \prod_{i=1}^{n} \Gamma\left(\frac{\alpha_{i}+1}{2}\right)}{2^{n} \pi^{n / 2} \Gamma\left(1+\left(n+\sum_{i=1}^{n} \alpha_{i}\right) / 2\right)} z^{\sum_{i} \alpha_{i} / 2} .
\end{aligned}
$$

Proof. In $\int_{S(z)} f(x) d x$, make the change of variable $x \mapsto y_{i}=x_{i}^{2}$ so that the $n$-ball $S(z)$ becomes the $n$-simplex $\Delta(z)$ in (2.1), and

$$
\int_{S(z)} f(x) d x=2^{-n} \int_{\Delta(z)} \prod_{i=1}^{n} y_{i}^{\left(\alpha_{i}-1\right) / 2} d y
$$

so that (3.3) follows from a direct application of Theorem 2.1.

To get the second equality in (3.3) it suffices to observe that $\operatorname{vol}(S(z))=$ $(z \pi)^{n / 2} / \Gamma(1+n / 2)$.

3.2. Exponentials of linear type. Now we consider the functions $f: \mathbb{R}^{n} \rightarrow$ $\mathbb{R}$ defined by

$$
x \mapsto f(x):=\mathrm{e}^{\langle c, x\rangle} .
$$

This is an important class of functions for it contains, in particular, the trigonometric polynomials when $c$ is allowed to be a purely imaginary complex vector.

Of course, since $f$ is analytic, one may consider its series expansion and apply Theorem 3.1 to each term. We will end up with a series expansion in $z$ for the integral over $S(z)$. However, the expansion in several dimensions can be quite heavy to perform. Alternatively, we may proceed directly to obtain the following. 
TheOREM 3.2. Let $f: \mathbb{R}^{n} \rightarrow \mathbb{R}$ be as in (3.4) and let $S(z)$ be as in (3.1). Then

$$
\int_{S(z)} f(x) d x=(z \pi)^{n / 2} \sum_{k=0}^{\infty} \frac{z^{k}\left(\sum_{i} c_{i}^{2} / 4\right)^{k}}{\Gamma(1+k+n / 2) k !}, \quad 0 \leq z<\infty .
$$

Alternatively,

$$
\int_{S(z)} f(x) d x=\operatorname{vol}(S(z))\left[1+\sum_{k=1}^{\infty} \frac{\left(\sum_{i} c_{i}^{2} / 2\right)^{k}}{k ! \prod_{j=1}^{k}(n+2 j)} z^{k}\right] .
$$

Proof. Let $g: \mathbb{R}^{+} \rightarrow \mathbb{R}$ be defined as follows:

$$
z \mapsto g(z):=\int_{S(z)} f(x) d x .
$$

Then $g(z)$ is well defined for all $z \in \mathbb{R}^{+}$because

$$
0<|g(z)|<\mathrm{e}^{z^{1 / 2} \max _{i}\left|c_{i}\right|} \operatorname{vol}(S(z))=\frac{(\pi z)^{n / 2}}{\Gamma(1+n / 2)} \mathrm{e}^{z^{1 / 2} \max _{i}\left|c_{i}\right|} .
$$

After a Fubini argument, its (one-sided) Laplace transform $G: \mathbb{C} \rightarrow \mathbb{C}$ reads

$$
G(\lambda)=\frac{1}{\lambda} \prod_{i=1}^{n}\left[\int_{-\infty}^{\infty} \mathrm{e}^{c_{i} x_{i}-\lambda x_{i}^{2}} d x_{i}\right], \quad \lambda \in \mathbb{C}, \Re(\lambda)>0,
$$

and we recognize in brackets the two-sided Laplace transform of $\mathrm{e}^{-\lambda x_{i}^{2}}$ evaluated at $-c_{i}$, that is,

$$
(\pi / \lambda)^{1 / 2} \cdot \mathrm{e}^{c_{i}^{2} /(4 \lambda)}
$$

Hence, we obtain

$$
G(\lambda)=\frac{1}{\lambda}\left(\frac{\pi}{\lambda}\right)^{n / 2} \mathrm{e}^{\sum_{i} c_{i}^{2} /(4 \lambda)} .
$$

Now we apply series inversion techniques inspired by those described in Widder [8, pp. 234-239]. The Laurent series expansion (around the origin) of the exponential $\mathrm{e}^{\beta / \lambda}=\sum_{k=0}^{\infty}(\beta / \lambda)^{k} / k$ ! allows us to write

$$
G(\lambda)=\pi^{n / 2} \sum_{k=0}^{\infty}\left[\frac{\left(\sum_{i} c_{i}^{2} / 4\right)^{k}}{k !} \cdot \frac{1}{\lambda^{1+k+n / 2}}\right] .
$$

Notice that the previous series converges uniformly on $\Re(\lambda) \geq 1$ so that we can apply the inverse Laplace transformation, term by term, to get the series on the right-hand side of (3.5).

Observe how fast the convergence of the series on the right-hand side of (3.5) is, in view of the coefficient $\Gamma(1+k+n / 2) k$ !. This fact allows us to conclude that the series converges uniformly on compact subsets of $\mathbb{R}^{+}$. 
Moreover, the series in (3.5) is a Bessel-type function for which a lot of tables are available in the literature. Finally, using the mean value theorem, we see that

$$
\int_{S(z)} f(x) d x=\operatorname{vol}(S(z)) f(\xi) \quad \text { for some } \xi \in S(z) .
$$

We can then obtain a series representation of $f(\xi)$ in terms of $z$ :

$$
f(\xi(z))=1+\sum_{k=1}^{\infty} \frac{\left(\sum_{i} c_{i}^{2} / 2\right)^{k}}{k ! \prod_{j=1}^{k}(n+2 j)} z^{k} .
$$

Trigonometric polynomials. We can handle trigonometric polynomials by letting $c \in \mathbb{C}$ be a purely imaginary vector $c:=a \sqrt{-1}$ with $a \in \mathbb{R}^{n}$, as we did in the case of integrating on a simplex $\Delta(z)$. However, since $\sin (\cdot)$ is an odd function, the integral of $x \mapsto \sin \langle a, x\rangle$ on the $n$-ball $S(z)$ vanishes. On the other hand, as a direct consequence of Theorem 3.2, we obtain

$$
\int_{S(z)} \cos \langle a, x\rangle d x=\operatorname{vol}(S(z))\left[1+\sum_{k=1}^{\infty} \frac{(-1)^{k}\left(\sum_{i} a_{i}^{2} / 2\right)^{k}}{k ! \prod_{j=1}^{k}(n+2 j)} z^{k}\right] .
$$

3.3. Exponentials of Gaussian type. We now consider the function $f$ : $\mathbb{R} \rightarrow \mathbb{R}$ with

$$
x \mapsto f(x):=\mathrm{e}^{-x^{\prime} Q x}
$$

for some real-valued positive definite symmetric matrix $Q \in \mathbb{R}^{n \times n}$.

THEOREM 3.3. Let $f: \mathbb{R}^{n} \rightarrow \mathbb{R}$ be as in (3.11) and $S(z)$ be as in (3.1). Let $\left\{\alpha_{i}\right\}$ be the strictly positive eigenvalues of $Q$. Then

$$
\int_{S(z)} f(x) d x=(z \pi)^{n / 2} \sum_{k=0}^{\infty} \frac{\beta_{k} z^{k}}{\Gamma(1+k+n / 2)}, \quad 0 \leq z<\infty,
$$

where the $\beta_{k}$ are the coefficients in the power series expansion around the origin of

$$
\prod_{i=1}^{n} \frac{1}{\sqrt{1+\alpha_{i} x}}=\sum_{k=0}^{\infty} \beta_{k} x^{k}, \quad|x|<\min \left\{1 / \alpha_{i}\right\} .
$$

Notice that we take the positive branch of all square roots in order to get $\prod_{i=1}^{n} 1 / \sqrt{1}=1$. Alternatively,

$$
\int_{S(z)} f(x) d x=\operatorname{vol}(S(z))\left[1+\sum_{k=1}^{\infty} \frac{\beta_{k}(2 z)^{k}}{\prod_{j=1}^{k}(n+2 j)}\right] .
$$

Proof. Following a previous argument, let $g: \mathbb{R}^{+} \rightarrow \mathbb{R}$ be defined as in (3.7). After a Fubini argument, its Laplace transform $G: \mathbb{C} \rightarrow \mathbb{C}$ reads 


$$
\begin{aligned}
G(\lambda) & =\frac{1}{\lambda} \int_{\mathbb{R}^{n}} \mathrm{e}^{-x^{\prime}(Q+\lambda I) x} d x=\frac{\pi^{n / 2}}{\lambda \operatorname{det}(\lambda I+Q)^{1 / 2}} \\
& =\frac{\pi^{n / 2}}{\lambda} \prod_{i=1}^{n}\left(\lambda+\alpha_{i}\right)^{-1 / 2} \\
& =\frac{\pi^{n / 2}}{\lambda^{1+n / 2}} \prod_{i=1}^{n}\left(1+\alpha_{i} / \lambda\right)^{-1 / 2}, \quad \lambda \in \mathbb{C}, \Re(\lambda)>0 .
\end{aligned}
$$

We take the positive branch of all square roots, so $\prod_{i=1}^{n} 1^{-1 / 2}=1$. Now we expand $\prod_{i=1}^{n}\left(1+\alpha_{i} / \lambda\right)^{-1 / 2}$ in a Laurent series, with $|\lambda|>\max \left\{\alpha_{i}\right\}$, to obtain

$$
G(\lambda)=\pi^{n / 2} \sum_{k=0}^{\infty} \frac{\beta_{k}}{\lambda^{1+k+n / 2}}, \quad \Re(\lambda)>\max \left\{\alpha_{i}\right\} .
$$

Notice that the above series converges uniformly on $\Re(\lambda) \geq 1+\max \left\{\alpha_{i}\right\}$, so that we can apply the inverse Laplace transformation, term by term, to get the series on the right-hand side of (3.12). Finally, (3.14) follows from $\operatorname{vol}(S(z))=(\pi z)^{n / 2} / \Gamma(1+n / 2)$.

We have to remark that, as in (3.9), the mean value theorem yields

$$
\int_{S(z)} f(x) d x=\operatorname{vol}(S(z)) f(\xi(z)), \quad \xi(z) \in S(z),
$$

with

$$
f(\xi(z))=1+\sum_{k=1}^{\infty} \frac{\beta_{k}(2 z)^{k}}{\prod_{j=1}^{k}(n+2 j)} .
$$

Finally, we analyse the general case of integrating the function

$$
x \mapsto f(x):=\mathrm{e}^{-x^{\prime} Q x+b^{\prime} x}, \quad x \in \mathbb{R}^{n},
$$

on an arbitrary ellipsoid $S$ centred at some point $a \in \mathbb{R}^{n}$. Recall that $Q$ is a real-valued positive definite symmetric matrix. We have already mentioned that $S(z)$ can be assumed to be an $n$-ball as in (3.1), by applying an affine transformation if necessary. Moreover, the expression $-x^{\prime} Q x+b^{\prime} x$ does not change its structure under an affine transformation, it remains of the form $-y^{\prime} Q_{0} y+b_{0}^{\prime} y+c$; and the constant $c$ can easily be ignored, since integrating $\mathrm{e}^{-y^{\prime} Q_{0} y+b_{0}^{\prime} y+c}$ reduces to integrating $\mathrm{e}^{c}$ times $\mathrm{e}^{-y^{\prime} Q_{0} y+b_{0}^{\prime} y}$.

Let $Q=V^{\prime} A V$ be the spectral decomposition of $Q$, where $A$ is the diagonal matrix whose entries $\alpha_{i}$ are the strictly positive eigenvalues of $Q$, and $V^{\prime} V=I$. 
TheOrem 3.4. Let $S(z)$ be as in (3.1) and $f: \mathbb{R}^{n} \rightarrow \mathbb{R}$ be as in (3.18). Then

$$
\int_{S(z)} f(x) d x=(z \pi)^{n / 2} \sum_{k=0}^{\infty} \frac{\beta_{k} z^{k}}{\Gamma(1+k+n / 2)}, \quad 0<z<\infty
$$

where the $\beta_{k}$ are the coefficients in the power series expansion around the origin of the following function (with $c:=V b / 2$ ):

$$
x \mapsto h(x):=\prod_{i=1}^{n} \frac{\mathrm{e}^{c_{i}^{2} x /\left(1+\alpha_{i} x\right)}}{\left(1+\alpha_{i} x\right)^{1 / 2}}, \quad|x|<\min \left\{1 / \alpha_{i}\right\} .
$$

Notice that we take the positive branch of all square roots in order to get $\prod_{i=1}^{n} 1^{-1 / 2}=1$. Alternatively,

$$
\int_{S(z)} f(x) d x=\operatorname{vol}(S(z))\left[1+\sum_{k=1}^{\infty} \frac{\beta_{k}(2 z)^{k}}{\prod_{j=1}^{k}(n+2 j)}\right] .
$$

Proof. Let $g: \mathbb{R}^{+} \rightarrow \mathbb{R}^{+}$be defined as in (3.7). Then its Laplace transform $G: \mathbb{C} \rightarrow \mathbb{C}$ reads as follows (we need to define $b_{0}=(Q+\lambda I)^{-1} b / 2$ and fix $\lambda \in \mathbb{C}$ with $\left.\Re(\lambda)>\max \left\{\alpha_{i}\right\}\right)$ :

$$
\begin{aligned}
G(\lambda) & =\frac{1}{\lambda} \int_{\mathbb{R}^{n}} \mathrm{e}^{-x^{\prime}(Q+\lambda I) x+b^{\prime} x} d x \\
& =\frac{1}{\lambda} \mathrm{e}^{b_{0}^{\prime}(Q+\lambda I) b_{0}} \int_{\mathbb{R}^{n}} \mathrm{e}^{-\left(x-b_{0}\right)^{\prime}(Q+\lambda I)\left(x-b_{0}\right)} d x \\
& =\frac{\pi^{n / 2}}{\lambda^{1+n / 2}} \mathrm{e}^{b_{0}^{\prime}(Q+\lambda I) b_{0}} \prod_{i=1}^{n}\left(1+\alpha_{i} / \lambda\right)^{-1 / 2}
\end{aligned}
$$

Moreover, setting $b=2 V^{\prime} c$ (or $\left.c=V b / 2\right)$, we obtain $b_{0}^{\prime}(Q+\lambda I) b_{0}=$ $c^{\prime}(A+\lambda I)^{-1} c$, and so

$$
G(\lambda)=\frac{\pi^{n / 2}}{\lambda^{1+n / 2}} \prod_{i=1}^{n} \frac{\mathrm{e}^{c_{i}^{2} /\left(\lambda+\alpha_{i}\right)}}{\left(1+\alpha_{i} / \lambda\right)^{1 / 2}}, \quad \Re(\lambda)>\max \left\{\alpha_{i}\right\} .
$$

The rest of the proof is similar to that of Theorem 3.3.

4. The general case on the simplex. In this section, we briefly outline a methodology valid for integrating functions $f: \mathbb{R}^{n} \rightarrow \mathbb{R}$ on the simplex $\Delta(z)$ of $(2.1)$. Let $g: \mathbb{R}^{+} \rightarrow \mathbb{R}$ be

$$
z \mapsto g(z):=\int_{\Delta(z)} f(x) d x
$$


Let $F: \mathbb{C}^{n} \rightarrow \mathbb{C}^{n}$ be the Laplace transform of $f$, that is,

$$
\lambda \mapsto F(\lambda):=\int_{\mathbb{R}_{+}^{n}} \mathrm{e}^{-\langle\lambda, x\rangle} f(x) d x, \quad \lambda \in D_{f} \subset \mathbb{C}^{n},
$$

where $D_{f}$ is the domain of definition of $F$. Recall that $D_{f}$ is empty when the function $f$ does not have a Laplace transform.

TheOREM 4.1. Let $f: \mathbb{R}^{n} \rightarrow \mathbb{R}$ be a function with Laplace transform $F: \mathbb{C}^{n} \rightarrow \mathbb{C}^{n}$, let $g$ be defined as in (4.1), and $H: \mathbb{C} \rightarrow \mathbb{C}$ be defined by $x \mapsto H(x):=F\left(x^{-1} e_{n}\right)$ with $e_{n}=(1, \ldots, 1)$ the unit vector in $\mathbb{R}^{n}$.

(i) Suppose there exist two real numbers $\varrho>-1$ and $r>0$ such that the function $H(x)$ can be expanded in a power series

$$
H(x)=\sum_{k=0}^{\infty} \beta_{k} x^{k+\varrho},
$$

which converges uniformly on the compact ball $B:=\{x \in \mathbb{C}:|x-r| \leq r\}$ of radius $r$ and centre at $r$. Notice that the intersection of $B$ and the real line is the closed interval $[0,2 r]$. Then

$$
g(z)=\sum_{k=0}^{\infty} \frac{\beta_{k}}{\Gamma(1+k+\varrho)} z^{k+\varrho}, \quad 0 \leq z<\infty .
$$

(ii) Assume that $g$ in (4.1) is bounded continuous. If there exist two real numbers $\varrho$ and $r>0$ such that $H$ can be expanded in a series

$$
H(x):=\sum_{k=0}^{\infty} \beta_{k} x^{-k-\varrho}, \quad x \in \mathbb{R}, x>0,
$$

where $\beta_{k}=O\left(r^{k} / k !\right)$ as $k \rightarrow \infty$, then

$$
g(z)=\sum_{k=0}^{\infty} \frac{\beta_{k}}{\Gamma(1-k-\varrho)} z^{-k-\varrho}, \quad r \mathrm{e}^{\pi}<x<\infty .
$$

Proof. Let $G: \mathbb{C} \rightarrow \mathbb{C}$ be the Laplace transform of $g$, that is,

$$
\begin{aligned}
G(\lambda) & =\int_{0}^{\infty} \mathrm{e}^{-\lambda z}\left[\int_{\Delta(z)} f(x) d x\right] d z=\int_{\mathbb{R}_{+}^{n}} f(x)\left[\int_{\sum_{i} x_{i}}^{\infty} \mathrm{e}^{-\lambda z} d z\right] d x \\
& =\frac{1}{\lambda} \int_{\mathbb{R}_{+}^{n}} \mathrm{e}^{-\lambda\left\langle e_{n}, x\right\rangle} f(x) d x=\frac{F\left(\lambda e_{n}\right)}{\lambda}=\frac{H(1 / \lambda)}{\lambda},
\end{aligned}
$$

where we have just applied Fubini's theorem. Now, in case (i), $G$ can be expanded in a series $G(\lambda)=\sum_{k=0}^{\infty} \beta_{k} \lambda^{-1-k-\varrho}$ which converges uniformly on $\Re(\lambda) \geq 1 /(2 r)$, so we can apply inverse Laplace transform, term by term, to get the series expansion of $g$ in (4.4). On the other hand, the series 
expansion of $g$ in (4.6) follows from the real series inversion techniques of Theorem 10.2 in Widder [8, pp. 236-239].

Once again, we remark that the original multivariate integration problem reduces to calculating a one-dimensional series expansion of the Laplace transform of $F$ evaluated at $x^{-1} e_{n}$. Moreover, notice that the results presented in Section 2 could have been deduced from Theorem 4.1. However, we have used the fact that $G$ could be inverted directly in several cases to get a closed form.

EXAMPLES. Here are some nontrivial examples in $\mathbb{R}^{2}$ that illustrate the efficiency of the approach presented in this section.

Let us recall the definitions of the Probability integral erf(·), Exponential integral function $\operatorname{Ei}(\cdot)$ and the Bessel function $J_{0}(\cdot)$ :

$$
\begin{array}{rlrl}
\operatorname{erf}(x) & :=\frac{2}{\sqrt{\pi}} \int_{0}^{x} \mathrm{e}^{-t^{2}} d t, & & x \in \mathbb{R}, x \geq 0, \\
\operatorname{Ei}(x) & :=\int_{-\infty}^{x} \frac{\mathrm{e}^{t}}{t} d t, & & x \in \mathbb{R}, \\
J_{0}(x):=\sum_{k=0}^{\infty} \frac{(-1)^{k}}{(k !)^{2}}\left(\frac{x}{2}\right)^{2 k}, & & x \in \mathbb{R} .
\end{array}
$$

\begin{tabular}{lcccc}
\hline & $f(x, y)$ & $F(u, v)$ & $G(\lambda)$ & $F\left(x^{-1} e_{2}\right)$ \\
\hline $\operatorname{Ex} 1$ & $\operatorname{erf}\left(\frac{x}{2 \sqrt{y}}\right)$ & $\frac{1}{(u+\sqrt{v}) u \sqrt{v}}$ & $\frac{1}{\lambda^{3}(1+\sqrt{\lambda})}$ & $\frac{x^{5 / 2}}{1+\sqrt{x}}$ \\
$\operatorname{Ex} 2$ & $\frac{\cos (2 \sqrt{a x y})}{\sqrt{\pi x}}$ & $\frac{\sqrt{u}}{u v+a}$ & $\frac{\sqrt{\lambda}}{\lambda\left(a+\lambda^{2}\right)}$ & $\frac{x^{3 / 2}}{1+a x^{2}}$ \\
$\operatorname{Ex} 3$ & $J_{0}(2 \sqrt{a x y})$ & $\frac{1}{u v+a}$ & $\frac{1}{\lambda\left(a+\lambda^{2}\right)}$ & $\frac{x^{2}}{1+a x^{2}}$ \\
$\operatorname{Ex} 4$ & $\frac{2 \sqrt{x y}}{\pi(x+y)}$ & $\frac{1}{(\sqrt{u}+\sqrt{v})^{2} \sqrt{u v}}$ & $\frac{1}{4 \lambda^{3}}$ & $\frac{x^{2}}{4}$ \\
$\operatorname{Ex} 5$ & $\frac{\sin (2 a \sqrt{x y})}{\pi a y}$ & $\frac{1}{u \sqrt{u v+a^{2}}}$ & $\frac{1}{\lambda^{2} \sqrt{a^{2}+\lambda^{2}}}$ & $\frac{x^{2}}{\sqrt{1+a^{2} x^{2}}}$ \\
$\operatorname{Ex6}$ & $-\frac{\mathrm{e}^{-x / y}}{y}$ & $\frac{\mathrm{e}^{v / u} \operatorname{Ei}(-v / u)}{u}$ & $\frac{\mathrm{e} \operatorname{Ei}(-1)}{\lambda^{2}}$ & $x \operatorname{eEi}(-1)$ \\
$\operatorname{Ex} 7$ & $\frac{1}{2 \sqrt{x^{2}+y^{2}}}$ & $\frac{1}{\sqrt{u^{2}+v^{2}}} \operatorname{arctanh} \frac{\sqrt{u^{2}+v^{2}}}{u+v}$ & $\frac{1}{\lambda^{2} \sqrt{2}} \operatorname{arctanh} \frac{1}{\sqrt{2}}$ & $\frac{x}{\sqrt{2}} \operatorname{arctanh} \frac{1}{\sqrt{2}}$ \\
\hline
\end{tabular}

Hence, for instance,

$$
\int_{\Delta(z)} \frac{2 \sqrt{x y}}{\pi(x+y)} d x d y=z^{2} / 4=\operatorname{vol}(\Delta(z)) \frac{1}{2} .
$$


Similarly,

$$
\int_{\Delta(z)} \frac{\sin (2 a \sqrt{x y})}{\pi a y} d x d y=z^{2} \sum_{k=0}^{\infty} \frac{a_{k} z^{k}}{(k+2) !}=2 \operatorname{vol}(\Delta(z)) \sum_{k=0}^{\infty} \frac{a_{k} z^{k}}{(k+2) !},
$$

where $\left\{a_{k}\right\}$ are the coefficients in the series

$$
\left(1+a^{2} z^{2}\right)^{-1 / 2}=\sum_{k=0}^{\infty} a_{k} z^{k}, \quad|z|<1 /|a| .
$$

\section{References}

[1] A. Barvinok, Computing the volume, counting integral points, and exponential sums, Discrete Comput. Geom. 10 (1993), 123-141.

[2] A. N. Podkorytov, Summation of multiple Fourier series over polyhedra, Vestnik Leningrad. Univ. Mat. Mekh. Astronom. 1980, no. 1, 51-58 (in Russian).

[3] J. B. Lasserre, Integration and homogeneous functions, Proc. Amer. Math. Soc. 127 (1999), 813-818.

[4] J. B. Lasserre and K. Avrachenkov, The multidimensional version of $\int_{a}^{b} x^{n} d x$, Amer. Math. Monthly 108 (2001), 151-154.

[5] F. Oberhettinger and L. Badii, Tables of Laplace Transforms, Springer, New York, 1973.

[6] A. H. Stroud, A fifth degree integration formula for the $n$-simplex, SIAM J. Numer. Anal. 6 (1969), 90-98.

[7] -, Approximate Calculation of Multiple Integrals, Prentice-Hall, Englewood Cliffs, NJ, 1971.

[8] D. V. Widder, An Introduction to Transform Theory, Academic Press, New York, 1971.

[9] O. C. Zienkiewicz and R. L. Taylor, The Finite Element Method, 4th ed., Vols. 1 and 2, McGraw-Hill, London, 1988.

\section{LAAS-CNRS}

7 Avenue du Colonel Roche 31077 Toulouse Cedex 4, France

E-mail: lasserre@laas.fr
Departamento de Matemáticas

CINVESTAV-IPN

Apdo. Postal 14740

México, D.F. 07000, Mexico

E-mail: eszeron@math.cinvestav.mx

Received on 13.9.2001;

revised version on 5.11.2001 